\title{
Rotor Dynamics Analysis of Rotating Machine Systems Using Artificial Neural Networks
}

\author{
M. Kalkat \\ Mechanical Engineering Department, Nigde University, Nigde, Turkey \\ Ş. Yıldırım and I. Uzmay \\ Mechanical Engineering Department, Erciyes University, Kayseri, Turkey
}

\begin{abstract}
A direct-coupled rotor system was designed to analyze the dynamic behavior of rotating systems in regard to vibration parameters. The vibration parameters are amplitude, velocity, and acceleration in the vertical direction. The system consisted of a machine analyzer, shaft, disk, master-trend software, and power unit. Four different points were detected and measured by the experimental setup. The vibration parameters were found and saved from master-trend software. These parameters were employed as the desired parameters of the network. A neural network is designed for analyzing a system's vibration parameters. The results showed that the network could be used as an analyzer of such systems in experimental applications.
\end{abstract}

Keywords Experimental setup, Neural network, Shaft, Vibration

Condition-monitoring techniques for the diagnosis of faults in rotating machinery need to be improved in order to be able to identify, as quickly as possible, the many different kinds of faults that can occur in a rotor-dynamic system. Vibration-response measurements yield a great deal of information concerning any faults in a rotating machine. The identification of common mass unbalance by vibration analysis is very well developed and can be performed in many ways; however, the identification of faults such as bowed or cracked shafts, rubbing, and bearing misalignment remains relatively basic. Much research must be applied to these areas so as to devise comprehensive fault diagnosis schemes that can automatically detect any faults that may arise

Received 25 June 2002; accepted 1 July 2002.

Address correspondence to M. Kalkat, Mechanical Engineering Department, Nigde University, Nigde, 51100, Turkey. E-mail: mkalkat@ nigde.edu.tr in a system and provide information concerning the best correction procedure to be used.

Recently, rotating machinery has been studied in greater detail. A thorough understanding of the principles of rotor dynamics is essential for engineers and scientists involved in the transportation and power-generation industries, as well as in many other fields on which we find ourselves relying to an increasing extent.

Because the analysis and design of rotating machinery are extremely critical in terms of the cost of both production and maintenance, it is not surprising that fault diagnosis of rotating machinery is a crucial aspect of the subject, one that is receiving ever more attention. As the design of rotating machinery becomes increasingly complex as a result of the rapid progress being made in technology, so must condition-monitoring strategies become more advanced in order to cope with the physical burdens being placed on the individual components of a machine. Modern condition-monitoring techniques encompass many different themes, one of the most important and informative being vibration analysis, a field in which much research has been carried out and a corresponding amount of literature produced. Using vibration analysis, the state of a machine can be constantly monitored, and detailed analyses may be made concerning the health of the machine and any faults that may be arising or may have already arisen, serious or otherwise. Common rotor-dynamic faults include self-excited vibration due to system instability and, more commonly, vibration due to some externally applied load, such as cracked or bent shafts or mass unbalance.

Vibration-condition monitoring as an aid to fault diagnosis was examined by Taylor (1995), who included information about the actual process of data analysis - how measured data should be processed in order for a diagnosis to be performed. Smalley and colleagues (1996) presented a method of assessing the severity of vibration in terms of the probability of damage by analyzing the vibration signals and ascertaining the relative cost 
of the damage using the net present value method. The question of whether to shut down a machine for maintenance was considered and some guidelines were formulated for comparing maintenance and downtime costs against the possible costs that would be incurred by damage.

By using the Pareto distribution method for machinery diagnostic tests, Cempel (1991) showed that the method he developed for the condition monitoring of tribovibroacoustical processes could be generalized for vibration processes and so used in vibration-condition monitoring. The reliability graph drawn from vibration measurements can easily be transformed into a life curve for a given machine, so the Pareto distribution lends itself to assessing both the condition of a machine and its residual time to breakdown. Su and Lin (1992) extended a previous vibration model proposed by McFadden and Smith (1984) to describe the bearing vibration caused by a single defect and provided detailed insight into the analysis of vibration spectra.

As technology advances, rotors become lighter and faster and tolerances become tighter. With this growing complexity it is important to eliminate as many sources of faults as is possible. New techniques are continually being developed to cope with the demand for fault-free machinery. For instance, Halliwell (1996) showed how it is possible to measure torsional vibration, which is important for the analysis of vibration in gears, using a laser torsional vibrometer, thus eliminating the need for cumbersome mechanical parts. The laser approach has many significant advantages in practical applications, where vibration measurement has previously caused problems. This is not only because of the practical difficulties of inserting measuring equipment, but also because extra mass and stiffness terms are often added to the system by the traditional methods.

Sekhar and Prabhu (1995) discussed the effect of a coupling misalignment on the vibration of rotating machinery. Shaft misalignment can be a major cause of vibration because of the reaction forces generated in the shaft couplings. It is generally accepted that a significant twofold vibration response is a major feature of bearing misalignment. A finite-element model of the rotor-coupling-bearing system was developed and the effect of misalignment was introduced through a coupling-coordinate system. The model agrees well with empirical results, in which the singlefold response is not affected nearly as significantly as the twofold response. By using this model it is therefore possible to predict the vibration response due to misalignment at the various harmonics; such a prediction is valuable in terms of both fault diagnosis and machinery design.

He and colleagues (1990) presented a new method of identifying rub failure between the rotating and stationary parts of a machine. The traditional method of spectrum analysis of rubbing is to measure the response spectra. A disadvantage of this method is, however, that the rub mechanism may produce noise in certain frequency bands, known as colored noise. The new method proposed - combining principal components and autoregressive spectra (PCAT) - was able to identify the chief characteristics of the colored noise. First an autocorrelation matrix was estimated on the basis of the vibration signal, and from that, the principal components were obtained by using the orthogonal transformation matrix of eigenvectors. The autoregressive modal coefficients and prediction error were then determined. Computer simulations with noisy data have shown that the PCAT method is an efficient tool for the identification of colored noise in rub failure in large rotating machinery. A thorough analysis of the transient response caused by rotor-stator interaction was given by Ghauri and colleagues (1996) in whose work the rubbing process was represented by a linear impact model (Coulomb friction). It was shown that under certain circumstances, sustained rotor-stator contact and reverse whirl could be delayed by asymmetry in the rotor support structure. A low coefficient of friction at the contact interface was also shown to delay reverse whirl. The issue of clearance effects on spiral vibrations due to rubbing was addressed by Childs and Jordan (1997), and it was shown that a clearance at the rub location improved the stability of the system, in particular with regard to unstable spiral vibrations.

For the diagnosis of anisotropy and asymmetry in rotating machinery, Lee and Joh (1994) developed a method of incorporating directional frequency response functions (dFRFs). Anisotropy and asymmetry may cause whirl, fatigue, and instability and can influence such system characteristics as unbalance and critical speeds. Complex modal testing was used to estimate the dFRFs. The example presented showed the proposed method to be very efficient in identifying anisotropy and asymmetry.

\section{THEORY OF THE ROTATING SYSTEM}

Let us consider the rotor shown in Figure 1 which by assumption rotates with a constant angular speed $\Omega$. The shaft $S$ of the rotor is supported rigidly at its ends. Assume that the shaft can be considered massless and flexible, whereas the element $E$ can be approximated by a particle of mass $m$. This particle is attached to the shaft at the center of gravity $G$ of the element $E$. The center of gravity $G$ is displaced by $\mu$ from the geometrical center of the shaft cross-section $C$. The distance $\mu$ represents the imbalance of the element $E$ and can be considered to be of small magnitude. To analyze motion of this system, let us introduce the inertial system of coordinates $X, Y$, and $Z$ as it is shown in Figure 2. The instantaneous position of the center $C$

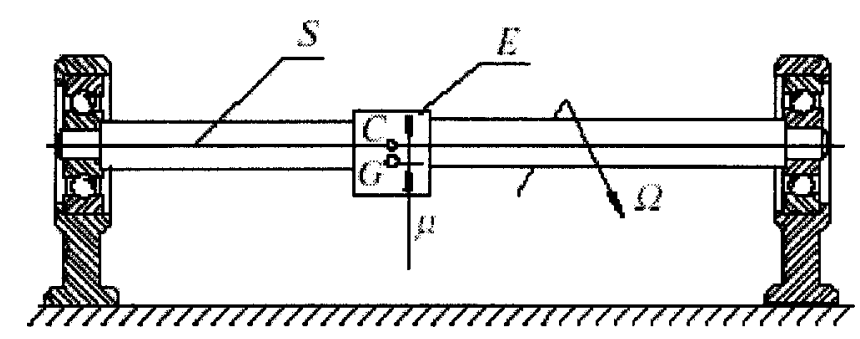

FIGURE 1

Description of the system. 


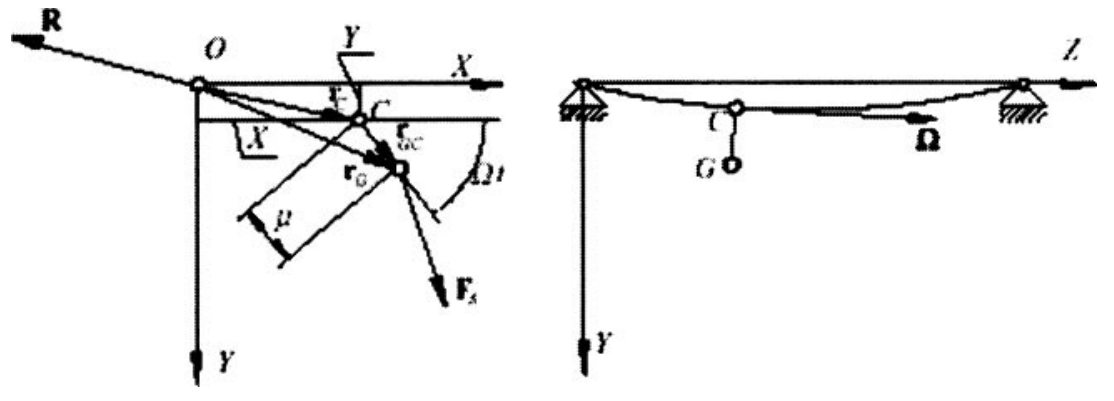

FIGURE 2

Representation of the absolute position of the center of gravity $G$.

is determined by the position vector $r_{C}$. The center of gravity $G$ rotates with respect to this center with the angular velocity $\Omega$. Since the angular velocity is constant, the relative instantaneous position of the center of gravity $G$ is determined by the angle $\Omega t$ and the imbalance $\mu$ (vector $r_{G C}$ ). The absolute position of the center of gravity $G$ in Figure 2 is denoted by $r_{G}$. The vector $F s$ represents the static resultant force acting on the element $E$. $R$ stands for the interaction force between the element considered and the shaft.

\section{MATHEMATICAL MODEL OF THE SYSTEM}

The motion of the center of gravity $G$ is governed by the Newton law

$$
m \ddot{r}_{G}=R+F_{s}
$$

where, according to Figure 2,

$$
\begin{aligned}
r_{G} & =I(X+\mu \cos \Omega t)+J(Y+\mu \sin \Omega t) \\
\mathrm{R} & =-I k X-J k Y \\
\mathrm{Fs} & =I F_{X s}+J F_{Y s}
\end{aligned}
$$

In the above formula, $k$ stands for the stiffness of the shaft at the point $C$, and $X$ and $Y$ are its coordinates.

The introduction of Equation (2) into Equation (1) results in the following set of differential equations:

$$
m\left(\ddot{Y}-\mu \Omega^{2} \sin \Omega t\right)=-k Y+F_{Y s}
$$

or, after reorganization,

$$
\begin{aligned}
m \ddot{X}+k X & =F_{X s}+m \mu \Omega^{2} \cos \Omega t \\
m \ddot{Y}+k Y & =F_{Y s}+m \mu \Omega^{2} \sin \Omega t
\end{aligned}
$$

The particular solution of the nonhomogeneous Equation (5),

$$
\begin{aligned}
m \ddot{X}+k X & =F_{X s} \\
m \ddot{Y}+k Y & =F_{Y s}
\end{aligned}
$$

yields the equilibrium position $\left(X_{S} ; Y_{S}\right)$. Upon assuming the particular position in the form

$$
\begin{gathered}
X=X s \\
Y=Y s
\end{gathered}
$$

one may obtain the following formulas for the coordinates of the equilibrium position, which are usually referred to as the static deflection of the shaft.

$$
X_{s}=\frac{F_{X s}}{k} \quad Y_{s}=\frac{F_{Y s}}{k}
$$

The total deflection of the shaft $X, Y$ is the sum of the static deflection $X_{s}, Y_{s}$, and the dynamic deflection $x, y$ (Figure 3):

$$
\begin{aligned}
& X=X s+\chi \\
& Y=Y s+y
\end{aligned}
$$

The introduction of Equation (8) into mathematical model in Equation (4) that govern the dynamic deflections $x y$

$$
m \ddot{y}+k y=m \mu \Omega^{2} \sin \Omega t
$$

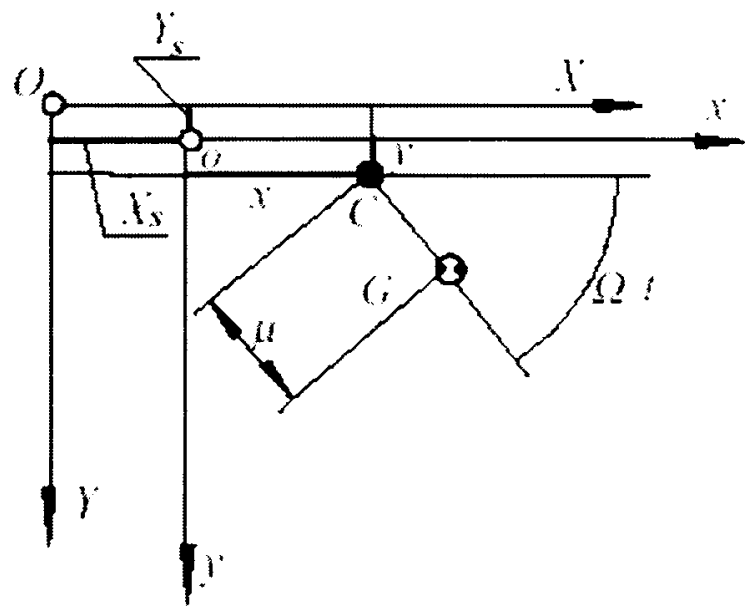

FIGURE 3

Total deflection of shaft on the planar surface. 
or

$$
\begin{gathered}
\ddot{x}+\omega^{2 \chi}=q \cos \Omega t \\
\ddot{y}+\omega^{2} y=q \sin \Omega t
\end{gathered}
$$

where

$$
w=\sqrt{\frac{k}{m}} \quad q=\mu \Omega^{2}
$$

Upon multiplying Equation (11) by the imaginary unit $i$ and adding Equations (10) and (11), one may obtain the equations of motion of the rotor in the following form:

$$
\ddot{z}+\omega^{2} z=q e^{i \Omega t}
$$

where

$$
z=\chi+i y
$$

The above equation governs motion of the rotor in the stationary system of coordinates $x, y$, and $z$.

Let us introduce the rotating system of coordinates $x_{R}, y_{R}$, and $z_{R}$ as shown in Figure 4. Axis $Z_{R}$ coincides axis $z$, and axes $x_{R}$ and $y_{R}$ rotate with the constant angular velocity $\Omega$. In terms of the complex notations, the position of the point $C$ in the stationary system of coordinates $x, y$, and $z$ is

$$
z=|z| e^{i \varphi}
$$

and in the rotating system of coordinates, it is

$$
z_{R}=\left|z_{R}\right| e^{i(\varphi-\Omega t)}=\left|z_{R}\right| e^{i \varphi} e^{-\Omega t}
$$

The introduction of Equation (15) into Equation (16) yields the relationship between coordinates of the same point in the stationary $(x, i y)$ and the rotating $\left(x_{R}, y_{R}\right)$ systems of coordinates:

$$
z_{R}=z e^{-i \Omega t}
$$

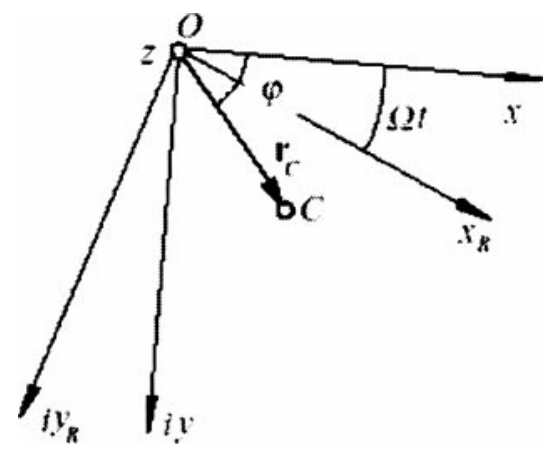

FIGURE 4

The rotating system coordinates.
The inverse transformation is

$$
z=z_{R} e^{+i \Omega t}
$$

Differentiating Equation (18) with respect to time, one can obtain

$$
\begin{aligned}
& \dot{z}=\dot{z}_{R} e^{i \Omega t}+z_{R} i \Omega e^{i \Omega t} \\
& \ddot{z}=\ddot{z}_{R} e^{i \Omega t}+2 \dot{z}_{R} i \Omega e^{i \Omega t}-z_{R} \Omega^{2} e^{+i \Omega t}
\end{aligned}
$$

The introduction of Equation (19) into mathematical model produces the equation of motion of the rotor in terms of the rotating system of coordinates:

$$
\ddot{z}_{R}+2 \dot{z}_{R} i \Omega+z_{R}\left(\omega^{2}-\Omega^{2}\right)=q
$$

\section{EXPERIMENTAL APPARATUS}

As can be seen in Figure 5, the system consisted of a shaft $2 \mathrm{~mm}$ in diameter and $27 \mathrm{~mm}$ long, with a $1000 \mathrm{~mm}$ length carrying a centrally located steel disk weighing $1.64 \mathrm{~kg}$. It was supported by identical rolling element bearings with UC 204 SKF ball bearings at the two ends and was driven by a $0.75 \mathrm{~kW}$, $230 \mathrm{v}$ AC electric motor, with a caplin, shaft-rotor system, bearing, frame body, Siemens Junior velocity control unit, and CSI Machine analyzer. First, experimental work was done to analyze the system's behavior without the load. Second, the system was analyzed using a load (the disk).

\section{ARTIFICIAL NEURAL NETWORKS}

Artificial neural networks (NNs) are made up of simple, highly interconnected processing units called neurons, each of which performs two functions: aggregation of its inputs from other neurons or the external environment and generation of an output based on the aggregated inputs. The output of a neuron is fed to other neurons to which it is connected via weighted links. Through this simple structure, neural networks (NNs) have been shown to be able to approximate most continuous functions, to any degree of accuracy, by choosing an appropriate number of neurons and output activation.

\section{Feedforward Neural Networks}

Feedforward NNs are made up of one or more hidden layers between the input and output layers, as illustrated in Figure 6. The functionality of the network is determined by specifying the strengths of the connection paths, called weights, and the neuron activation function. The input layer distributes inputs to the first hidden layer. The inputs then propagate forward through the network and each neuron computes its output according to the equation

$$
x_{i}^{m}=g\left(\sum_{j=1}^{n_{m-1}} W_{i j}^{m} x_{j}^{m-1}+b_{i}^{m}\right)
$$




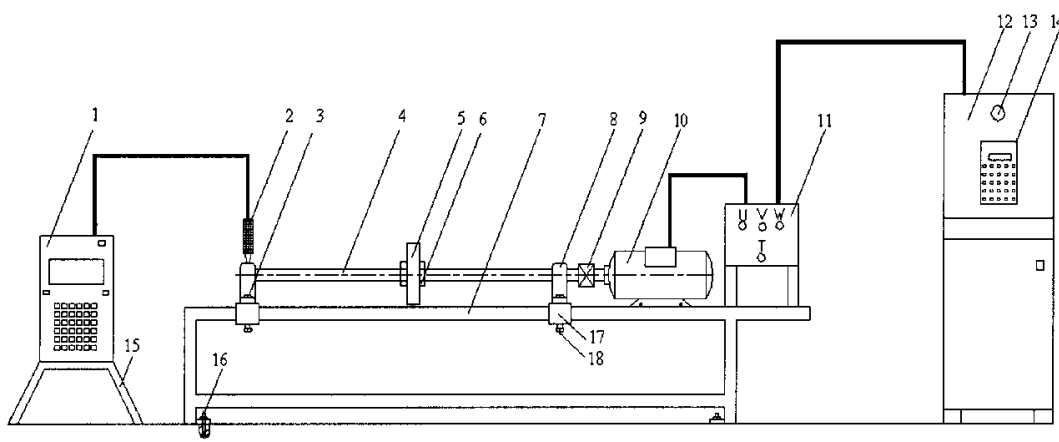

\begin{tabular}{|l|l|l|l|}
\hline 1 & CSt 2110 Machine Analyzer & 10 & ACEl ektric Motor \\
\hline 2 & Acceleration Meter & 11 & Component Table \\
\hline 3 & M16 Nut & 12 & Control Panel \\
\hline 4 & Shaft & 13 & Fuse \\
\hline 5 & Disc & 14 & ACVelocity Control Unit \\
\hline 6 & M27x2 Nut & 15 & Tabie \\
\hline 7 & Table & 16 & M 10 Concrete Nut \\
\hline 8 & Bearing & 17 & Connection Component \\
\hline 9 & Caphin & 18 & M 16 Nut \\
\hline
\end{tabular}

FIGURE 5

Representation of the experimental apparatus.

where $x_{i}^{m}$ is the output of the $i$ th neuron in the $m$ th layer, $W_{i j}^{m}$ is the weight of the connection between the $j$ th neuron of the $(m-1)$ th layer and the $i$ th neuron of the $m$ th layer, and $b_{i}^{m}$ is the bias of the $i$ th neuron in the $m$ th layer. $b_{i}^{m}$ can be regarded as the weight of the connection between a fixed input of unit value and neuron $i$ in layer $m$. The function $g(\cdot)$ is called the neuron activation function. The argument

$$
z_{i}^{m}=\sum_{j=1}^{n_{m-1}} W_{i j}^{m} x_{j}^{m-1}+b_{i}^{m}
$$

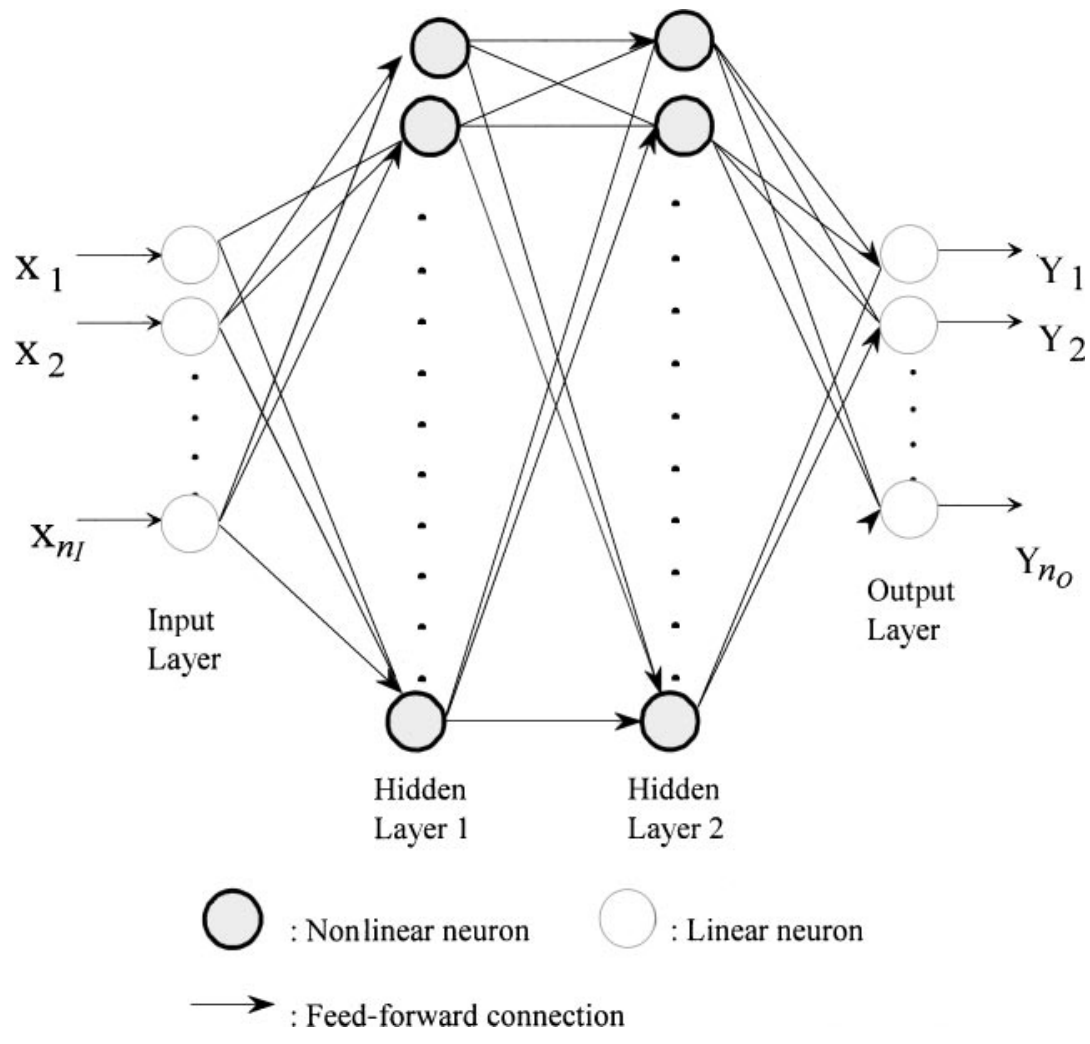

FIGURE 6

A layered feed-forward artificial neural network, Funahashi (1989). 
is the activation for the $i$ th neuron in the $m$ th layer. The function $g(\cdot)$ is assumed to be differentiable and to have a strictly positive first derivative.

For neurons in the hidden layers, the activation function is often chosen to be

$$
g(z)=\frac{1}{1+e^{-z}}
$$

Because the activation is nonlinear, the neurons are said to be nonlinear neurons. Because in system modeling applications, the dynamic range of the output data may be greater than 1 , the activation function of the output nodes is chosen to be linear, and the output nodes are said to be linear neurons. Thus, the $i$ th output node performs a weighted sum of its inputs as follows:

$$
y_{i}=\sum_{j=1}^{n_{m-1}} W_{i j}^{O} x_{j}^{m-1}
$$

where $m$ represents the output layer and $\mathrm{W}_{i j}^{O}$ is the weight of the connection between the $j$ th neuron of the last hidden layer and the $i$ th neuron of the output layer.

Only networks with one hidden layer are considered in the present study because the results of Funahashi (1989) and Funahashi (1989) show that this is sufficient to approximate all continuous functions. Let $\Theta=\left[\theta_{1} \ldots \ldots \ldots \theta_{n}\right]^{T}$ represent all the unknown weights and the biases of the network, where $n$ denotes the dimension of the parameter vector $\Theta$ and is defined as $n=n_{H}\left(n_{I}+1\right)+n_{H} n_{O}$, where $n_{I}, n_{H}$, and $n_{O}$ refer to the number of neurons in the input layer, in the hidden layer, and in the output layer, respectively. The $i$ th output of a network with a single layer of hidden units can then be defined by

$$
\begin{array}{r}
y_{i=1} \sum_{j=1}^{n} W_{i j}^{O} x_{j}^{H}=\sum_{j=1}^{n} W_{i j}^{O} g\left(\sum_{k=1}^{{ }^{n} I} W_{j k}^{I} x_{k}^{I}+b_{j}^{H}\right) \\
1 \leq i \leq n_{O}
\end{array}
$$

where $\mathbf{x}^{I}=\left[x_{1}^{I} \ldots \ldots \ldots x_{n I}^{I}\right]^{T}$ is the input vector to the network; $W_{j k}^{I}$ is the weight of the connection between the $k$ th neuron of the input layer and the $j$ th neuron of the hidden layer; and $b_{j}^{H}$ is the bias of the $j$ th neuron in the hidden layer.

\section{EXPERIMENTAL AND NEURAL NETWORK RESULTS}

Experimental work was carried out on the proposed experimental apparatus. The system, as represented earlier, was analyzed with a disk on the shaft. Four different points were marked on the frame for measuring the vibration parameters of the system at different working speeds.

Two types of NNs were employed to predict the vibration of the system. The structural and training parameters of the NN are given in Table 1. First, the shaft speed was $150 \mathrm{rpm}$ for two types of the network. Table 2 indicates the accuracy of the network for different shaft speeds. Shaft speed was increased and the
TABLE 1

Shaft Speeds and Training Parameters of the Neural Networks

\begin{tabular}{ccccr}
\hline $\begin{array}{c}\text { Shaft speed } \\
(\mathrm{rpm})\end{array}$ & NN type & $\eta$ & $\alpha$ & \multicolumn{1}{c}{$N$} \\
\hline 150 & NN1 & 0.1 & 0 & 150000 \\
150 & NN2 & 0.1 & 0 & 150000 \\
300 & NN1 & 0.1 & 0 & 150000 \\
300 & NN2 & 0.1 & 0 & 150000 \\
450 & NN1 & 0.1 & 0 & 150000 \\
450 & NN2 & 0.1 & 0 & 150000 \\
900 & NN1 & 0.1 & 0 & 150000 \\
900 & NN2 & 0.1 & 0 & 150000 \\
1050 & NN1 & 0.1 & 0 & 2784 \\
1050 & NN2 & 0.1 & 0 & 68566 \\
1200 & NN1 & 0.1 & 0 & 150000 \\
1200 & NN2 & 0.1 & 0 & 150000 \\
1350 & NN1 & 0.1 & 0 & 18281 \\
1350 & NN2 & 0.1 & 0 & 24352 \\
\hline
\end{tabular}

NN, neural network.

amplitude of the system in the vertical direction was measured up to critical speeds of the shaft. As indicated in Table 2, the neural predictor exactly follows the experimental results of the system for the critical speed of $1050 \mathrm{rpm}$ by the shaft. At the speed of $1200 \mathrm{rpm}$, the vibration amplitude of the shaft is very small and is acceptable. The neural predictor can be employed in the experimental model of the system at this working speed. The hidden neuron size plays an important role during the training of the network. When size of the neuron was increased, the

TABLE 2

RMSE and Percent Correct for Various Types of Networks and Shaft Speeds

\begin{tabular}{cccc}
\hline $\begin{array}{c}\text { Shaft speed } \\
(\mathrm{rpm})\end{array}$ & NN type & RMSEs & $\begin{array}{r}\text { Percent correct } \\
(\%)\end{array}$ \\
\hline 150 & NN1 & 0.09057 & 84 \\
150 & NN2 & 0.09000 & 86 \\
300 & NN1 & 0.05711 & 82 \\
300 & NN2 & 0.06258 & 92 \\
450 & NN1 & 0.04700 & 94 \\
450 & NN2 & 0.04407 & 92 \\
900 & NN1 & 0.03056 & 98 \\
900 & NN2 & 0.03389 & 96 \\
1050 & NN1 & 0.03380 & 100 \\
1050 & NN2 & 0.03353 & 100 \\
1200 & NN1 & 0.04255 & 96 \\
1200 & NN2 & 0.05876 & 86 \\
1350 & NN1 & 0.03550 & 100 \\
1350 & NN2 & 0.03498 & 96 \\
\hline
\end{tabular}

NN, neural network; RMSE, rotating machine system errors. 
training time of the network increased. Ten neurons may be used to achieve greater accuracy by the predictor.

\section{CONCLUSIONS}

This investigation illustrates the effectiveness of the artificial $\mathrm{NN}$ predictor for vibration analysis of a rotor-bearing system with a disk. The predictor was found to identify the faults to a good degree of accuracy. It has been found that the testing success of the network depends on the learning, momentum terms, and neuron size in the hidden layer. Finally, the network systems could be used as an analyzer for rotating-machinery systems in experimental applications.

\section{REFERENCES}

Cempel, C. 1991. Condition evolution of machinery and its assessment from passive diagnostic experiment. Mechanical Systems and Signal Processing 5:317-326.

Childs, D. W., and Jordan, L. T. 1997. Clearance effects on spiral vibrations due to rubbing. Proceedings of the ASME Design Engineering Technical Conference, Paper DETC97/VIB-4058.

Ding, J., and Krodkiewski, J. M. 1993. Inclusion of static indetermination in the mathematical model for nonlinear dynamic analyses of multi-bearing rotor systems. Journal of Sound and Vibration 164:267-280.

Flack, R. D., Rooke, J. H., Bielk, J. R., and Gunter, E. J. 1982. Comparison of the unbalance responses of Jeffcott rotors with shaft bow and shaft runout. Journal of Mechanical Design Transactions of the ASME 104:318-328.

Frank, P. M. 1994. Enhancement of robustness in observer-based faultdetection. International Journal of Control 59:955-981.

Frank, P. M., and Köppen-Seliger, B. 1997. New developments using artificial intelligence in fault diagnosis. Engineering Applications of Artificial Intelligence 10:3-14.

Garcia, E. A., and Frank, P. M. 1997. Deterministic nonlinear observerbased approaches to fault diagnosis: A survey. Control Engineering Practice 5:663-670.

Gasch, R. 1976. Dynamic behaviour of a simple rotor with a crosssecional crack. Proceedings of the Institution of Mechanical Engineers: Vibrations in Rotating Machinery 178/76:123-128.

Gasch, R. 1993. A survey of the dynamic behavior of a simple rotating shaft with a transverse crack. Journal of Sound and Vibration 160:313-332.

Genta, G. 1993. Vibration of Structures and Machines: Practical Aspects. New York: Springer-Verlag.

Ghauri, M. K. K., Fox, C. H. J., and Williams, E. J. 1996. Transient response and contact due to sudden imbalance in a flexible rotorcasing system with support asymmetry. Proceedings of the Institution of Mechanical Engineers: Vibrations in Rotating Machinery 383394. Oxford: Institution of Mechanical Engineers.

Halliwell, N. A. 1996. The laser torsional vibrometer: a step forward in rotating machinery diagnostics. Journal of Sound and Vibration 190:399-418.

He, Z. J., Sheng, Y. D., and Qu, L. S. 1990. Rub failure signature analysis for large rotating machinery. Mechanical Systems and Signal Processing 4:417-424.

Howell, J. 1994. Model-based fault-detection in information poor plants. Automatica 30:929-943.
Huang, S. C., Huang, Y. M., and Shieh, S. M. 1993. Vibration and stability of a rotating shaft containing a transverse crack. Journal of Sound and Vibration 162:387-401.

Isermann, R. 1993. Fault diagnosis of machines via parameter estimation and knowledge processing: tutorial paper. Automatica 29:815835.

Isermann, R. 1994. On the applicability of model-based fault-detection for technical processes. Control Engineering Practice 2:439450.

Isermann, R. 1997. Supervision, fault detection and fault-diagnosis methods: an introduction. Control Engineering Practice 5:639_ 652.

Isermann, R., and Ballé, P. 1997. Trends in the application of modelbased fault detection and diagnosis of technical processes. Control Engineering Practice 5:709-719.

Jun, O. S., Eun, H. J., Earmme, Y. Y., and Lee, C. W. 1992. Modeling and vibration analysis of a simple rotor with a breathing crack. Journal of Sound and Vibration 155:273-290.

Krodkiewski, J. M., Ding, J., and Zhang, N. 1994. Identification of unbalance change using a nonlinear mathematical-model for multibearing rotor systems. Journal of Sound and Vibration 169:685698.

Lee, C. W., and Joh, C. Y. 1994. Development of the use of directional frequency-response functions for the diagnosis of anisotropy and asymmetry in rotating machinery: theory. Mechanical Systems and Signal Processing 8:665-678.

Lees, A. W., and Friswell, M. I. 1997. The evaluation of rotor unbalance in flexibly mounted machines. Journal of Sound and Vibration 208:671-683.

Leonhardt, S., and Ayoubi, M. 1997. Methods of fault diagnosis. Control Engineering Practice 5:683-692.

Loukis, E., Mathioudakis, K., and Papailiou, K. 1994. Optimizing automated gas-turbine fault-detection using statistical patternrecognition. Journal of Engineering for Gas Turbines and Power: Transactions of the ASME 116:165-171.

McFadden, P. D., and Smith, J. D. 1984. Model for the vibration produced by a single defect in a rolling element bearing. Journal of Sound and Vibration 96:69-92.

Meng, G., and Hahn, E. J. 1997. Dynamic response of a cracked rotor with some comments on crack detection. Journal of Engineering for Gas Turbines and Power: Transactions of the ASME 119:447455.

Morton, P. G. 1985. Modal balancing of flexible shafts without trial weights. Proceedings of the Institution of Mechanical Engineers, Part C: Mechanical Engineering Science 199:71-78.

Natke, H. G., and Cempel, C. 1991. Fault detection and localisation in structures: a discussion. Mechanical Systems and Signal Processing 5:345-356.

Neale, M. J. 1995. The Tribology Handbook. London: ButterworthHeinemann.

Parkinson, A. G. 1991. Balancing of rotating machinery. Proceedings of the Institution of Mechanical Engineers, Part C: Mechanical Engineering Science 205:53-66.

Parkinson, A. G., Darlow, M. S., and Smalley, A. J. 1984. Balancing flexible rotating shafts with an initial bend. AIAA Journal 22:683689.

Patton, R. J., and Chen, J. 1997. Observer-based fault detection and isolation: robustness and applications. Control Engineering Practice 5:671-682. 
Seibold, S., and Weinert, K. 1996. A time domain method for the localization of cracks in rotors. Journal of Sound and Vibration 195: $57-73$.

Sekhar, A. S., and Prabhu, B. S. 1995. Effects of coupling misalignment on vibrations of rotating machinery. Journal of Sound and Vibration 185:655-671.

Smalley, A. J., Baldwin, R. M., Mauney, D. A., and Millwater, H. R. 1996. Towards risk-based criteria for rotor vibration. Proceedings of the Institution of Mechanical Engineers: Vibrations in Rotating Machinery 517-527. Oxford: United Kingdom.

Srinivasan, A., and Batur, C. 1994. Fault-detection and isolation in an unsupervised learning environment. Pattern Recognition Letters $15: 235-242$.
Su, Y. T., and Lin, S. J. 1992. On initial fault detection of a tapered roller bearing: frequency-domain analysis. Journal of Sound and Vibration 155:75-84.

Tan, S. G., and Wang, X. X. 1993. A theoretical introduction to lowspeed balancing of flexible rotors: unification and development of the modal balancing and influence coefficient techniques. Journal of Sound and Vibration 168:385-394.

Taylor, J. I. 1995. Back to the basics of rotating machinery vibration analysis. Sound and Vibration 29:12-16.

Wang, X. F., and McFadden, P. D. 1996. Simulation models for bearing vibration generation and fault detection via neural networks. Proceedings of the Institution of Mechanical Engineers: Vibrations in Rotating Machinery 441-450. Oxford: United Kingdom. 

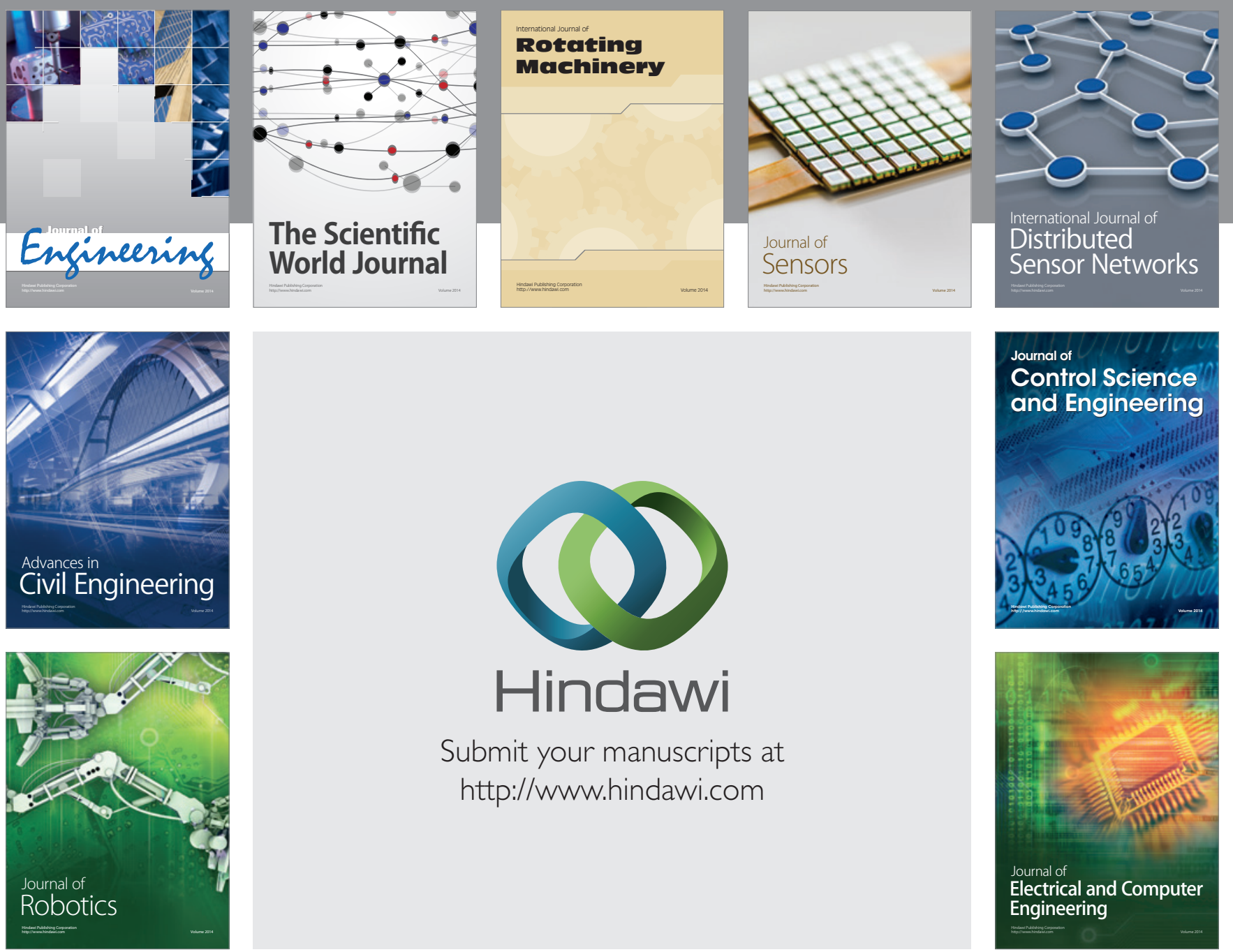

Submit your manuscripts at

http://www.hindawi.com
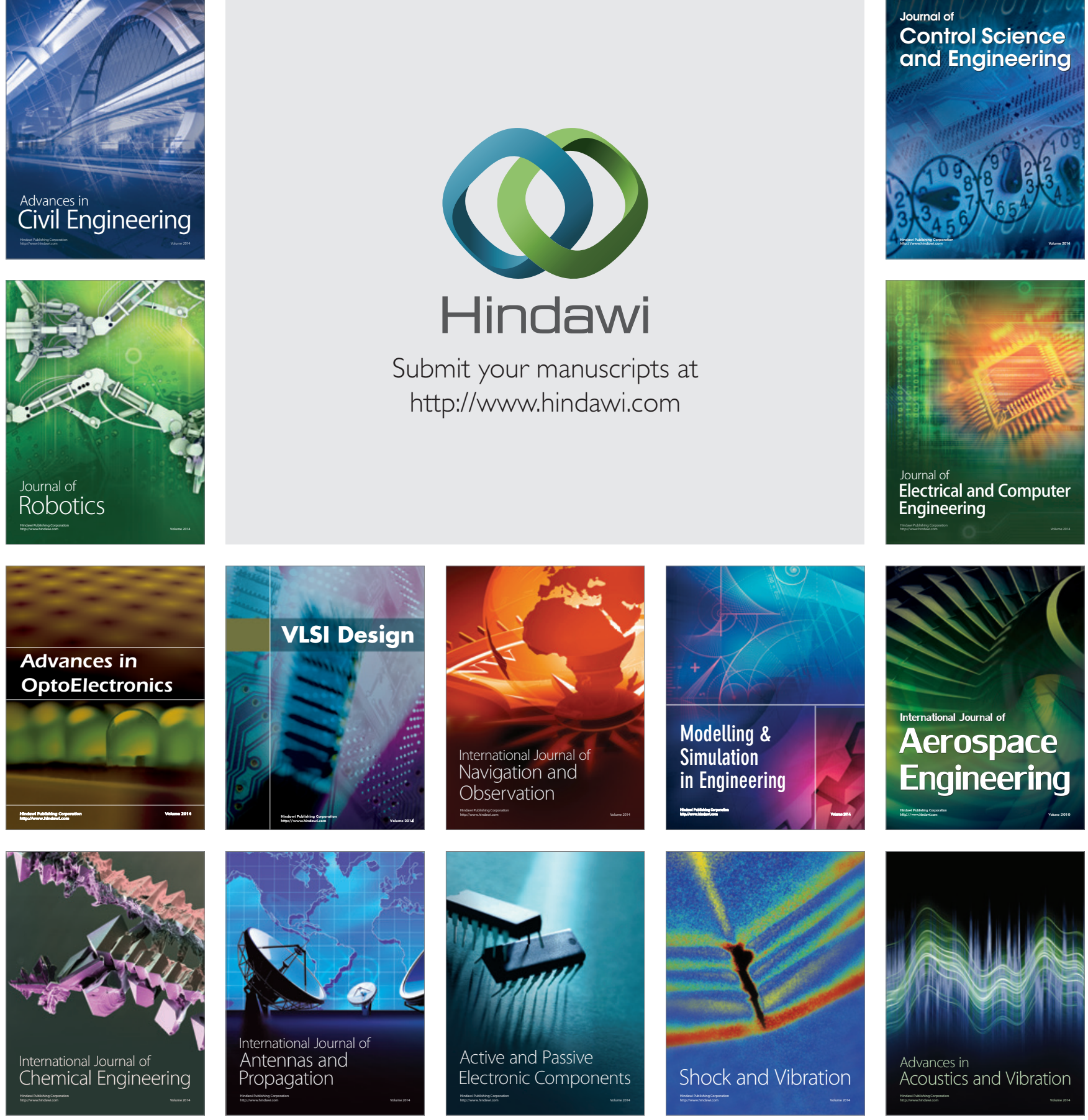